\title{
NNLO predictions for Z-boson pair production at the LHC
}

\section{G. Heinrich, S. Jahn, S.P. Jones, M. Kerner and J. Pires}

Max Planck Institute for Physics, Föhringer Ring 6, München, 80805 Germany

E-mail: gudrun@mpp.mpg.de, sjahn@mpp.mpg.de, sjones@mpp.mpg.de, kerner@mpp.mpg.de, pires@mpp.mpg.de

ABSTRACT: We present a calculation of the NNLO QCD corrections to Z-boson pair production at hadron colliders, based on the $\mathrm{N}$-jettiness method for the real radiation parts. We discuss the size and shape of the perturbative corrections along with their associated scale uncertainties and compare our results to recent LHC data at $\sqrt{s}=13 \mathrm{TeV}$.

Keywords: NLO Computations, QCD Phenomenology

ARXIV EPRINT: 1710.06294 


\section{Contents}

1 Introduction 1

2 Details of the calculation $\quad 2$

2.1 Discussion of the IR subtraction procedure 5

$\begin{array}{llr}3 & \text { Results } & 7\end{array}$

4 Conclusions $\quad 12$

\section{Introduction}

The pair production of $Z$-bosons at the LHC is an important process to test the electroweak sector of the Standard Model (SM). It is sensitive to anomalous gauge boson couplings and constitutes an irreducible background to the production of a Higgs boson decaying into vector bosons and to New Physics searches.

Recent measurements already include a combined ATLAS and CMS study of anomalous triple gauge couplings in $Z Z$ production based on Run I data [1], as well as measurements at $8 \mathrm{TeV}[2-4]$ and $13 \mathrm{TeV}[5-9]$.

The NLO QCD corrections to $Z$-boson pair production were calculated first for stable $Z$-bosons in refs. $[10,11]$, and later including leptonic decays in the narrow-width approximation in ref. [12]. Leptonic decays including spin correlations and off-shell effects have been taken into account in refs. [13, 14].

$Z$-boson pair production via gluon fusion is suppressed by two powers of the strong coupling compared to the $q \bar{q}$ channel, but contributes significantly to the total cross section due to the large gluon flux at the LHC. The one-loop calculation for stable $Z$-bosons has been performed in refs. [15, 16], leptonic decays and off-shell effects have been included and studied in refs. [17-24]. Soft gluon resummation to the signal/background interference process $g g\left(\rightarrow H^{(*)}\right) \rightarrow Z Z$ also has been considered [25].

Recently, the 2-loop amplitudes for $q \bar{q} \rightarrow V V^{\prime}[26-28]$ and $g g \rightarrow V V^{\prime}[29,30]$ became available, and led to the calculation of the NNLO corrections for $Z$-boson pair production, for on-shell $Z$-bosons [31] as well as including leptonic decays [32]. The two-loop corrections to the gluon fusion channel were also calculated $[33,34]$ and even combined with a parton shower in ref. [35].

Electro-weak (EW) NLO corrections were first calculated for stable vector bosons [3638], and including decays within the Herwig++ framework [39]. Very recently, NLO EW corrections including full off-shell effects have become available [40-42].

The calculation in refs. $[31,32]$ is based on the $q_{T}$-subtraction scheme [43] for the doubly unresolved real radiation occurring at NNLO. In this letter, we report on the 


\begin{tabular}{|ll|}
\hline NNLO contributions & perturbative order \\
\hline $0 \rightarrow q Z Z g g \bar{q}$ & tree-level \\
$0 \rightarrow q Z Z Q \bar{Q} \bar{q}$ & tree-level \\
\hline $0 \rightarrow q Z Z g \bar{q}$ & one-loop \\
\hline $0 \rightarrow g g Z Z$ & one-loop \\
$0 \rightarrow q \bar{q} Z Z$ & two-loop \\
\hline
\end{tabular}

Table 1. Perturbative order of the matrix elements for $Z Z$ production at NNLO.

calculation of the NNLO corrections to on-shell $Z$-boson pairs using a different method, based on $N$-jettiness subtraction [44, 45]. The effect of massive quark loops has been estimated to be at the level of a permille contribution to the total cross section in ref. [31]. However, calculations performed in an $s / m_{t}^{2}$ expansion framework [46, 47] indicate that the contributions may be larger, and they certainly will be important in the region of large values of the 4-lepton invariant mass $m_{4 l}$, which is sensitive to the coupling of the longitudinal $Z$-boson components to the top quarks loops.

\section{Details of the calculation}

The NNLO computation requires the evaluation of the tree-level scattering amplitudes with two additional partons (double-real (RR) contribution), of the one-loop amplitudes with one additional parton (real-virtual (RV) contribution) and the two-loop corrections to the Born process (double-virtual (VV) contribution). In this way we systematically combine all the amplitudes containing two additional powers in the strong coupling constant with respect to the Born process such that the final result is NNLO accurate in perturbation theory. In table 1 we list the matrix elements for $Z Z$ production at NNLO.

Although the sum of virtual and real corrections yields a finite result, the individual contributions contain singularities of infrared (IR) and ultraviolet nature, such that a direct numerical evaluation is not possible. Virtual and real corrections come from phase space integrals of different multiplicity; therefore a framework to combine them must be such that the divergent regions in the real-radiation contribution (corresponding to soft and collinear emissions which map to configurations with one or two particles less, and therefore are degenerate with the virtual contribution) can be extracted and cancelled with the singularities of the virtual matrix elements.

In this work we employ the $N$-jettiness subtraction scheme $[44,45,48,49]$ to perform the evaluation of the NNLO cross section. We begin by reviewing the definition of the $N$-jettiness variable introduced in refs. [50, 51],

$$
\tau_{N}=\frac{2}{Q^{2}} \sum_{k} \min \left\{q_{a} \cdot p_{k}, q_{b} \cdot p_{k}, q_{1} \cdot p_{k}, \ldots, q_{N} \cdot p_{k}\right\},
$$

where $N$ denotes the number of jets desired in the final state and the sum runs over all QCD radiated particles. In eq. (2.1) the $q_{a}, q_{b}$ and $q_{1}, \ldots, q_{N}$ are a fixed set of massless 
reference momenta for the two beam jets and the $N$ observed jets, the $p_{k}$ are the parton momenta, and the dimensionful parameter $Q^{2}$ is the hard interaction scale. For the specific case of a colourless diboson system in the final state, eq. (2.1) reduces to the 0-jettiness or beam thrust which in the leptonic frame reads [50, 52],

$$
\mathcal{T}_{0}=Q \tau_{0}=\sum_{k} \min \left\{e^{Y_{Z Z}} n_{a} \cdot p_{k}, e^{-Y_{Z Z}} n_{b} \cdot p_{k}\right\}
$$

where $n_{a}=(1,0,0,1)$ and $n_{b}=(1,0,0,-1)$ define the beam axis and the $p_{k}$ are defined in the hadronic centre-of-mass frame. In the context of $N$-jettiness subtractions, taking into account the boost with rapidity $Y_{Z Z}$ of the Born system ensures that the power corrections are independent of $Y_{Z Z}$ [52].

Looking at the definition of 0 -jettiness in eq. (2.2) we can observe that $\mathcal{T}_{0} \rightarrow 0$ in the limit where the QCD emission $p_{k}$ is soft or collinear to the initial state. For this reason values of $\mathcal{T}_{0}$ close to zero indicate a final state containing the $Z Z$ pair and only IR (soft and collinear) emissions. In this way the $N$-jettiness variable can be used as a slicing parameter in any real-radiation phase space integral to separate infrared singular regions from hard and resolved configurations. In that sense the approach extends the slicing methods developed in the early 90's to compute higher-order corrections at NLO [53] to NNLO.

To proceed we employ a $\mathcal{T}_{0}^{\text {cut }}$ in the real-radiation NNLO phase space and split the cross section into regions above and below $\mathcal{T}_{0}^{\text {cut }}[44,45,49]$,

$$
\begin{aligned}
\sigma_{\mathrm{NNLO}}= & \int \mathrm{d} \Phi_{N}\left|\mathcal{M}_{V V}\right|^{2}+\int \mathrm{d} \Phi_{N+1}\left|\mathcal{M}_{R V}\right|^{2} \theta_{0}^{<} \\
& +\int \mathrm{d} \Phi_{N+2}\left|\mathcal{M}_{R R}\right|^{2} \theta_{0}^{<}+\int \mathrm{d} \Phi_{N+1}\left|\mathcal{M}_{R V}\right|^{2} \theta_{0}^{>} \\
& +\int \mathrm{d} \Phi_{N+2}\left|\mathcal{M}_{R R}\right|^{2} \theta_{0}^{>} \\
\equiv & \sigma_{\mathrm{NNLO}}\left(\mathcal{T}_{0}<\mathcal{T}_{0}^{\text {cut }}\right)+\sigma_{\mathrm{NNLO}}\left(\mathcal{T}_{0}>\mathcal{T}_{0}^{\text {cut }}\right) .
\end{aligned}
$$

In eq. (2.3) we have abbreviated $\theta_{0}^{<}=\theta\left(\mathcal{T}_{0}^{\text {cut }}-\mathcal{T}_{0}\right)$ and $\theta_{N}^{>}=\theta\left(\mathcal{T}_{0}-\mathcal{T}_{0}^{\text {cut }}\right)$, and have suppressed any (infrared-safe) measurement function under the phase space integral. The first three terms in this expression all have $\mathcal{T}_{0}<\mathcal{T}_{0}^{\text {cut }}$, and are collectively denoted as $\sigma_{\mathrm{NNLO}}\left(\mathcal{T}_{0}<\mathcal{T}_{0}^{\text {cut }}\right)$, while the remaining two terms, with $\mathcal{T}_{0}>\mathcal{T}_{0}^{\text {cut }}$, are collectively denoted as $\sigma_{\mathrm{NNLO}}\left(\mathcal{T}_{0}>\mathcal{T}_{0}^{\text {cut }}\right)$. Contributions with Born-level kinematics necessarily have $\mathcal{T}_{0}=0 .{ }^{1}$ Contributions with $\mathcal{T}_{0}>\mathcal{T}_{0}^{\text {cut }}$ necessarily contain one or more well separated hadronic energy deposits and thus reproduce the $Z Z+$ jet cross section at NLO. The contributions with $\mathcal{T}_{0}<\mathcal{T}_{0}^{\text {cut }}$ correspond to the limit of the $Z Z+$ jet NLO cross section where the jet is unresolved. The key advantage that allows the computation of the cross section at NNLO below $\mathcal{T}_{0}^{\text {cut }}$ is the fact that in the limit where all QCD emission is soft or collinear,

\footnotetext{
${ }^{1}$ Prior to its application for fixed-order perturbative QCD calculations a similar partitioning of the phase space was introduced by the GENEvA collaboration [54,55] in the context of merging fixed-order calculations with parton showers.
} 
the cross section can be approximately computed using the machinery of Soft-Collinear Effective Theory (SCET) [56]. In particular, the existence of a factorization theorem that gives an all-orders description of $N$-jettiness for small $\mathcal{T}_{N}$ less than some value $\mathcal{T}_{N}^{\text {cut }}$ allows the cross section to be written in the schematic form,

$$
\sigma\left(\mathcal{T}_{N}<\mathcal{T}_{N}^{\text {cut }}\right)=\int H \otimes B \otimes B \otimes S \otimes\left[\prod_{n}^{N} J_{n}\right]+\cdots,
$$

where $H$ describes the effect of hard radiation from the purely virtual corrections to the process, $B$ encodes the effect of radiation collinear to one of the two initial beam directions, $S$ describes soft radiation and $J_{n}$ contains the radiation collinear to hard final-state jets. The ellipsis denote power-suppressed terms which become negligible for $\mathcal{T}_{N} \ll Q$.

We have expanded the formula in eq. (2.4) to second order in the strong coupling constant to obtain the $\sigma_{\mathrm{NNLO}}\left(\mathcal{T}_{0}<\mathcal{T}_{0}^{\text {cut }}\right)$ cross section for $Z Z$ production at hadron colliders. In particular this includes contributions from the universal quark beam function at two loops [57] and the 0-jettiness soft function at two-loops [58, 59]. The process dependent hard function has been extracted from the two-loop amplitude computed in ref. [28] via an interface to the program qqvvamp. We do not include massive top-quark loops in the $q \bar{q} Z Z$ two-loop amplitude. Using $N_{f}=5$ therefore introduces the chiral anomaly stemming from subdiagrams where one $Z$-boson and two gluons are attached to a $b$-quark triangle. However, we neglect this anomalous contribution in our calculation as the anomaly must cancel once the top quark loops are included, following the same strategy as advocated in ref. [33].

In SCET renormalised form, the IR finite one- and two-loop amplitudes can be written at renormalisation scale $\mu^{2}$ as $[60,61]$

$$
\begin{aligned}
& \Omega_{N}^{(1), \text { finite }}=\Omega^{(1)}-I_{1}^{N}(\epsilon) \Omega^{(0)}, \\
& \Omega_{N}^{(2), \text { finite }}=\Omega^{(2)}-I_{1}^{N}(\epsilon) \Omega^{(1)}-I_{2}^{N}(\epsilon) \Omega^{(0)},
\end{aligned}
$$

where the $N$-jettiness subtraction operators are defined by ${ }^{2}$

$$
\begin{aligned}
& I_{1}^{N}(\epsilon)=\frac{\Gamma_{0}^{\prime}}{8 \epsilon^{2}}+\frac{\boldsymbol{\Gamma}_{0}}{4 \epsilon}, \\
& I_{2}^{N}(\epsilon)=-\frac{\left(\Gamma_{0}^{\prime}\right)^{2}}{128 \epsilon^{4}}-\frac{6 \beta_{0} \Gamma_{0}^{\prime}+2 \Gamma_{0}^{\prime} \boldsymbol{\Gamma}_{0}}{64 \epsilon^{3}}-\frac{8 \beta_{0} \boldsymbol{\Gamma}_{0}+2\left(\boldsymbol{\Gamma}_{0}\right)^{2}-\Gamma_{1}^{\prime}}{64 \epsilon^{2}}+\frac{\boldsymbol{\Gamma}_{1}}{16 \epsilon},
\end{aligned}
$$

with

$$
\begin{array}{ll}
\Gamma_{0}^{\prime}=-2 C_{F} \gamma_{0}^{\text {cusp }}, & \Gamma_{1}^{\prime}=-2 C_{F} \gamma_{1}^{\text {cusp }}, \\
\boldsymbol{\Gamma}_{0}=-C_{F} \gamma_{0}^{\text {cusp }} \log \left(\frac{\mu^{2}}{-s}\right)+2 \gamma_{0}^{q}, & \boldsymbol{\Gamma}_{1}=-C_{F} \gamma_{1}^{\text {cusp }} \log \left(\frac{\mu^{2}}{-s}\right)+2 \gamma_{1}^{q},
\end{array}
$$

\footnotetext{
${ }^{2}$ The subtraction operators are identical to those given for diphoton production in appendix A, eq. (A.17) of ref. [62], but these formulae appear to contain two typographical errors. Specifically, we find that $\Gamma_{0}^{\prime}$ should be multiplied to $\beta_{0}$ in the second term of eq. (2.6) and that the last term has a factor 16 in the denominator.
} 
and constants

$$
\begin{aligned}
\gamma_{0}^{\text {cusp }} & =4, \quad \gamma_{1}^{\text {cusp }}=\left(\frac{268}{9}-\frac{4 \pi^{2}}{3}\right) C_{A}-\frac{80}{9} T_{F} n_{f}, \quad \gamma_{0}^{q}=-3 C_{F} \\
\gamma_{1}^{q} & =\left(-\frac{3}{2}+2 \pi^{2}-24 \zeta_{3}\right) C_{F}^{2}+\left(-\frac{961}{54}-\frac{11 \pi^{2}}{6}+26 \zeta_{3}\right) C_{F} C_{A}+\left(\frac{130}{27}+\frac{2 \pi^{2}}{3}\right) C_{F} T_{F} n_{f} \\
\beta_{0} & =\frac{11}{6} C_{A}-\frac{4}{6} T_{F} n_{f}, \quad C_{A}=N, \quad C_{F}=\frac{N^{2}-1}{2 N}, \quad T_{F}=\frac{1}{2}
\end{aligned}
$$

In ref. [28] the finite remainder of the one- and two-loop form factors for vector boson pair production are provided in the $q_{T^{-}}$[63] and Catani- [64] subtraction schemes. By comparing the definition of the subtraction schemes, the form factors in the $N$-jettiness scheme can be derived from those of the $q_{T}$-scheme according to,

$$
\begin{aligned}
& \Omega_{N}^{(1), \text { finite }}=\Omega_{q_{T}}^{(1), \text { finite }}+\Delta I_{1} \Omega_{q_{T}}^{(0), \text { finite }}, \\
& \Omega_{N}^{(2), \text { finite }}=\Omega_{q_{T}}^{(2), \text { finite }}+\Delta I_{1} \Omega_{q_{T}}^{(1), \text { finite }}+\Delta I_{2} \Omega_{q_{T}}^{(0), \text { finite }},
\end{aligned}
$$

with the scheme conversion coefficients given by

$$
\begin{aligned}
\Delta I_{1}= & \frac{\pi^{2}}{12} C_{F} \\
\Delta I_{2}= & \frac{\pi^{4}}{288} C_{F}^{2}+\left(-\frac{607}{162}+\frac{67 \pi^{2}}{144}+\frac{77 \zeta_{3}}{36}-\frac{\pi^{4}}{72}+\frac{11 i \pi^{3}}{72}\right) C_{A} C_{F} \\
& +\left(\frac{41}{81}-\frac{5 \pi^{2}}{72}-\frac{7 \zeta_{3}}{18}-\frac{i \pi^{3}}{36}\right) C_{F} n_{f},
\end{aligned}
$$

where, for brevity, we have set $\mu^{2}=s$.

Finally we have obtained the $\sigma_{\mathrm{NNLO}}\left(\mathcal{T}_{0}>\mathcal{T}_{0}^{\text {cut }}\right)$ contribution of the $Z Z$ NNLO cross section using the tree level matrix elements from VBFNLO $[65,66]$ for the double-real emission phase space integral cross-checked with MadGraph5 [67], while the one-loop amplitudes for the real-virtual phase space were generated with GoSam [68, 69] and cross-checked with OpenLoops [70]. GoSam uses QGRAF [71], FORM [72] and Spinney [73] for the generation of the Feynman diagrams, and offers a choice from Samurai [74], golem95C [75-77] and Ninja [78, 79] for the reduction. At run time the amplitudes were computed using Ninja, which calls OneLOop [80] for the master integrals, and rescued using an implementation of Ninja in quadruple precision for unstable phase space points. We also include the loop induced one-loop squared corrections in the $g g \rightarrow Z Z$ channel, which are formally of NNLO accuracy, keeping full dependence on the top quark mass and on the Higgs mediated contributions using GoSam.

\subsection{Discussion of the IR subtraction procedure}

Before we present our numerical results for $Z Z$ production at hadron colliders we would like to make a few remarks concerning the IR subtraction scheme employed for this calculation. As mentioned in the previous section, the $N$-jettiness subtraction scheme is a non-local 
subtraction scheme. In local subtraction schemes the IR divergent phase space integrals are regulated by the introduction of suitable IR real-radiation counterterms that satisfy two basic properties,

- reproduce locally, for each phase space point in a singular region, the physical IR divergent soft and collinear limits of the matrix elements of the process under consideration;

- be simple enough to allow their analytic integration and generate a local and pointwise analytic pole cancellation between the explicit $1 / \epsilon$-poles of the virtual corrections and the $1 / \epsilon$-poles of the integrated real-radiation counterterms.

Some flexibility however exists on how the singular limits are locally subtracted and how the real-radiation phase space is parametrised. For antenna subtraction [81-83] physical matrix elements with three partons [84] at tree-level and one-loop suffice to reproduce single unresolved limits in QCD amplitudes, while four parton antennae [85, 86] subtract doubly unresolved configurations at NNLO [87-90]. Examples of local IR subtraction schemes which employ a structured decomposition of the real-radiation phase space based on singular IR limits of QCD amplitudes [91-94] have also been developed and applied to specific NNLO calculations. For the specific case of colourless systems in the initial state local subtractions have been developed in refs. [95, 96]. The extension of the $N$-jettiness method towards local subtractions has also been conceptually discussed in ref. [45].

On the other hand, non-local IR subtraction schemes regulate the singularities of the real-radiation phase space integrals by the introduction of a suitable variable $(N$-jettiness or the transverse momentum $q_{T}$ of a colourless system for $q_{T}$-subtraction $[43]^{3}$ ) that regulates the phase space integration by separating IR divergent regions from hard and resolved ones according to eq. (2.3). In this way contributions to the cross section for $\mathcal{T}_{0}$ above and below $\mathcal{T}_{0}^{\text {cut }}$ are separately logarithmically divergent. However, in the sum all the logarithmic dependence on $\mathcal{T}_{0}^{\text {cut }}$ should cancel, provided the value of $\mathcal{T}_{0}^{\text {cut }}$ employed in the phase space integration is small enough such that the SCET approximation to the cross section is valid. In particular, for each $1 / \epsilon$-IR pole in dimensional regularisation there is a corresponding logarithmically divergent coefficient predicted from SCET in the $\mathcal{T}_{0} \rightarrow 0$ limit, according to

$$
\frac{1}{\epsilon^{n}} \sim \log ^{n}\left(\frac{\mathcal{T}_{0}}{\mu}\right)
$$

The infrared pole cancellation in this case is observed through the cancellation between the universal and analytically known terms predicted by SCET, integrated over the Born phase space, and the Monte Carlo integration over the real-radiation phase space of the real-emisson matrix elements for small $\mathcal{T}_{0}$. The method of $N$-jettiness meanwhile has been applied successfully to various processes calculated at NNLO [44, 48, 49, 62, 98-104].

Due to the non-local IR subtraction method employed in our calculation of the NNLO corrections we found it necessary to do the following optimisations at the Monte Carlo

\footnotetext{
${ }^{3}$ An extension of $q_{T}$-subtraction to colourful final states has been worked out in ref. [97].
} 
integration level in order to observe the independence of our results on the choice of the slicing parameter value $\mathcal{T}_{0}^{\text {cut }}$ :

- introduce a phase space generator where the 0 -jettiness variable $\mathcal{T}_{0}$ is directly sampled by VEGAS. This can be achieved by applying the transformation

$$
p_{ \pm, i}=E_{i} \pm p_{z, i}, \quad \mathrm{~d} E_{i} \mathrm{~d} p_{z, i}=\frac{1}{2} \mathrm{~d} p_{+, i} \mathrm{~d} p_{-, i}
$$

to the integration over the real radiation phase space. ${ }^{4}$ With the momenta defined in the center-of-mass system of the $Z$-bosons, the 0 -jettiness as defined in eq. (2.2) then reads

$$
\mathcal{T}_{0}=\min \left(p_{+, 1}, p_{-, 1}\right)+\min \left(p_{+, 2}, p_{-, 2}\right) .
$$

With further transformations, where the regions with $p_{+, i}<p_{-, i}$ and $p_{+, i}>p_{-, i}$ have to be distinguished, it is then possible to directly sample $\mathcal{T}_{0}$, followed by the sampling of $\min \left(p_{+, 1}, p_{-, 1}\right)$, which also fixes the value of $\min \left(p_{+, 2}, p_{-, 2}\right)$. Afterwards, the two missing values of $p_{ \pm, i}$ can be sampled.

- have a fast implementation of the double-real and real-virtual matrix elements for $Z Z$-production which is stable in the multiple soft and collinear limits.

The first optimisation ensures that the real-radiation phase space generator properly samples the phase space boundaries determined by the choice of slicing parameter $\mathcal{T}_{0}^{\text {cut }}$ and that the phase space integral converges. The second optimisation ensures that the matrix elements are fast and stable enough to be integrated near the singular IR limits which give the bulk of the cross section for the $\left(\mathcal{T}_{0}>\mathcal{T}_{0}^{\text {cut }}\right)$ phase space integrals when $\mathcal{T}_{0}^{\text {cut }}$ is small. This is in contrast with a local subtraction scheme, where the real radiation subtraction terms ensure that the integrand vanishes as we approach a singular region.

\section{Results}

Our numerical studies for proton-proton collisions at centre-of-mass energy $\sqrt{s}=13 \mathrm{TeV}$ are for on-shell $Z$-boson pair production. We use the MSTW2008 [105] and NNPDF-3.0 [106] sets of parton distribution functions via the LHAPDF [107] interface, with densities and $\alpha_{s}$ evaluated at each corresponding order (i.e. we use $(n+1)$-loop $\alpha_{s}$ at $\mathrm{N}^{n} \mathrm{LO}$, with $n=0,1,2$ ) and we consider $N_{f}=5$ massless quark flavours. The default renormalisation $\left(\mu_{R}\right)$ and factorisation $\left(\mu_{F}\right)$ scales are set to $\mu_{R}=\mu_{F}=m_{Z}$. We use the $G_{\mu}$ EW scheme where the EW input parameters have been set to $G_{F}=1.16639 \times 10^{-5}, m_{W}=80.399 \mathrm{GeV}$ and $m_{Z}=91.1876 \mathrm{GeV}$. The top quark and Higgs boson masses that are included in the RV one-loop contributions and in the loop-induced $g g$ channel have been set to $m_{t}=173.2 \mathrm{GeV}$ and $m_{H}=125 \mathrm{GeV}$, respectively. We do not include top quark contributions in the double virtual two-loop diagrams.

\footnotetext{
${ }^{4}$ For each transformation, the integration boundaries, which we do not state explicitly, have to be changed accordingly.
} 


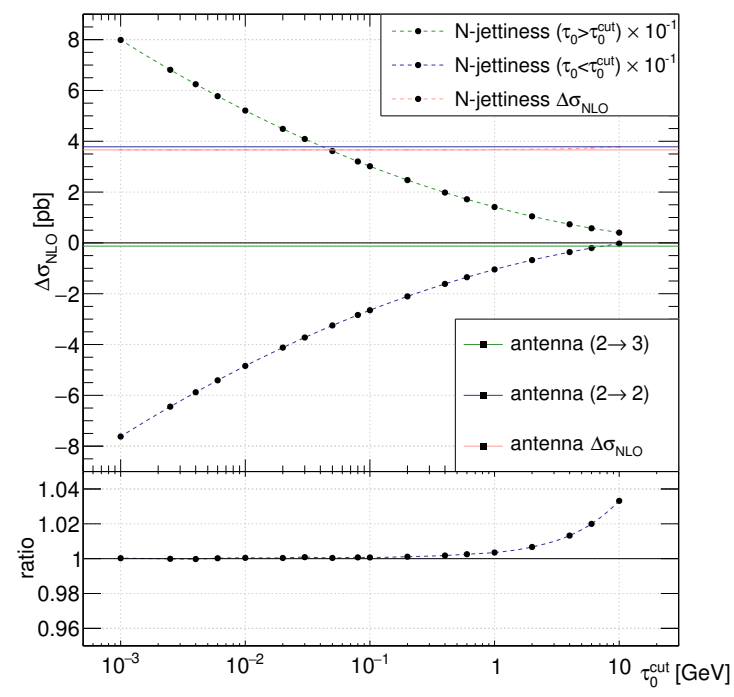

(a)

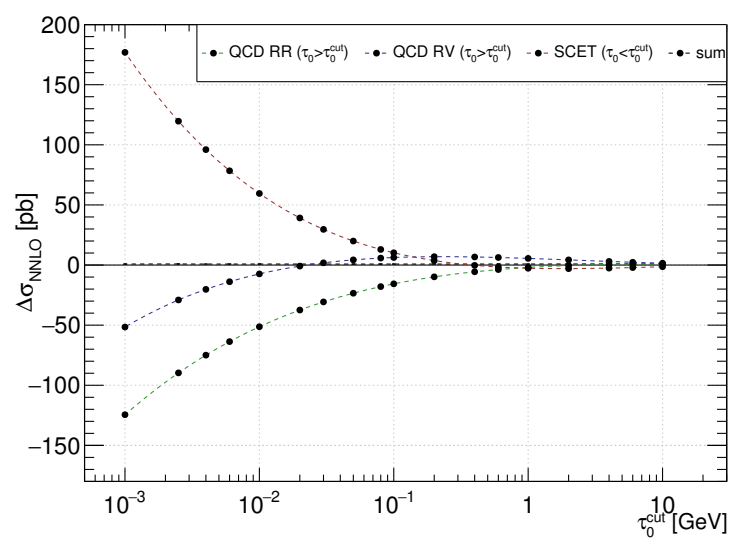

(b)

Figure 1. (a) NLO coefficient to the inclusive $Z Z$ cross section computed with $N$-jettiness subtraction (dashed-lines) and antenna subtraction (solid lines) as a function of $\mathcal{T}_{0}^{\text {cut }}$. We show separately the contributions of the $2 \rightarrow 3$ and $2 \rightarrow 2$ phase space integrals for antenna subtraction, and the $\sigma_{\mathrm{NLO}}\left(\mathcal{T}_{0}>\mathcal{T}_{0}^{\text {cut }}\right)$ and $\sigma_{\mathrm{NLO}}\left(\mathcal{T}_{0}<\mathcal{T}_{0}^{\text {cut }}\right)$ phase space integrals for $N$-jettiness. The ratio plot shows $\Delta \sigma_{\mathrm{NLO}}\left(N\right.$-jettiness) over $\Delta \sigma_{\mathrm{NLO}}($ antenna). (b) NNLO coefficient to the inclusive $Z Z$ cross section computed with $N$-jettiness subtraction (dashed lines) as a function of $\mathcal{T}_{0}^{\text {cut }}$. We show the separate cross sections for $\sigma_{\mathrm{NNLO}}\left(\mathcal{T}_{0}>\mathcal{T}_{0}^{\text {cut }}\right)$ from the double-real and real-virtual phase space integrals and for $\sigma_{\mathrm{NNLO}}\left(\mathcal{T}_{0}<\mathcal{T}_{0}^{\text {cut }}\right)$ from the SCET phase space integrals together with their sum.

We show in figure 1 the NLO and NNLO coefficients of the $Z Z$ cross section as a function of $\mathcal{T}_{0}^{\text {cut }}$. On the left-hand side we compare the NLO results obtained using either antenna subtraction or $N$-jettiness subtraction and observe full agreement in the evaluation of the NLO corrections $\Delta \sigma_{\mathrm{NLO}}$. We present separate results for the phase space integration with real emission kinematics $(2 \rightarrow 3)$ and Born-like kinematics $(2 \rightarrow 2)$. Obviously, using a local subtraction scheme (antenna subtraction), all phase space integrals contributing to $\Delta \sigma_{\mathrm{NLO}}$ are independent of the choice of $\mathcal{T}_{0}^{\text {cut }}$. Moreover, the bulk of the NLO coefficient comes from the $2 \rightarrow 2$ phase space integral which determines where more statistical precision is needed to obtain an accurate result. On the other hand, using $N$-jettiness we observe that both phase space integrals are separately double-logarithmically divergent and therefore a very good numerical precision is needed for both contributions to improve the accuracy of the final result. In figure $1 \mathrm{~b}$ we present the NNLO coefficient of the $Z Z$ cross section as a function of $\mathcal{T}_{0}^{\text {cut }}$. In this case we observe that the phase space integrals for the contributions $\sigma_{\mathrm{NNLO}}\left(\mathcal{T}_{0}<\mathcal{T}_{0}^{\text {cut }}\right)$ and $\sigma_{\mathrm{NNLO}}\left(\mathcal{T}_{0}>\mathcal{T}_{0}^{\text {cut }}\right)$ are logarithmically divergent to the fourth power in $\log \left(\mathcal{T}_{0}^{\text {cut }}\right)$, and for typical values of $\mathcal{T}_{0}^{\text {cut }}$ in the range $10^{-2}-10^{-3} \mathrm{GeV}$ need to be known with better than permille level accuracy to achieve an accurate determination of the NNLO coefficient.

In order to study in more detail the independence of the NNLO coefficient on the choice of slicing parameter $\mathcal{T}_{0}^{\text {cut }}$ we present in figure 2 on a smaller scale the NNLO coefficient 


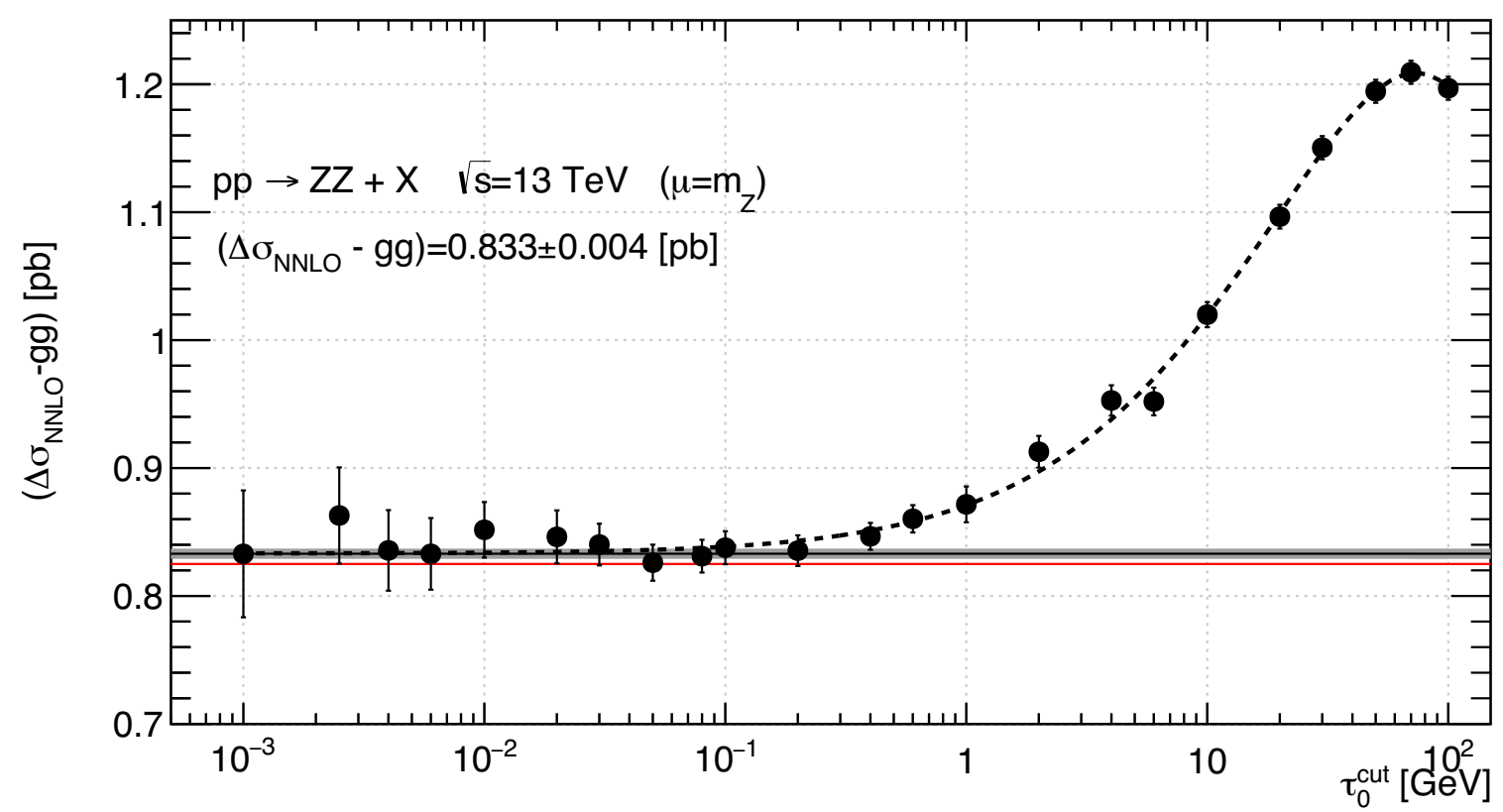

Figure 2. $\mathcal{T}_{0}^{\text {cut }}$ dependence of the NNLO coefficient for $Z Z$ production with the $\mathcal{T}_{0}$ independent $g g \rightarrow Z Z$ contribution subtracted. The black dashed line shows the fit of the $\mathcal{T}_{0}^{\text {cut }}$ dependence of the NNLO coefficient (black data points) to the analytic form in equation (3.1). The $\mathcal{T}_{0}^{\text {cut }} \rightarrow 0$ limit is shown as a solid black line with a grey band showing the uncertainty on the fitted parameter. The red line represents the NNLO coefficient reconstructed from the NNLO result obtained in ref. [31].

after combining the contributions for $\sigma_{\mathrm{NNLO}}\left(\mathcal{T}_{0}<\mathcal{T}_{0}^{\text {cut }}\right)$ and $\sigma_{\mathrm{NNLO}}\left(\mathcal{T}_{0}>\mathcal{T}_{0}^{\text {cut }}\right)$ as black data points. Within the errors we observe a plateau in the region $\mathcal{T}_{0}^{\text {cut }}=10^{-1} \sim 10^{-3} \mathrm{GeV}$ where the results tend to a constant. In addition we can observe for larger values of $\mathcal{T}_{0}^{\text {cut }}$ $\left(\mathcal{T}_{0}^{\text {cut }}>10^{-1} \mathrm{GeV}\right)$ the on-set of the power corrections to the $N$-jettiness SCET factorisation theorem which we do not compute. The fact that the on-set of power corrections shows up for fairly large values of $\mathcal{T}_{0}^{\text {cut }}$ with respect to other processes $[44,49,102]$ seems to indicate that for $Z Z$ production their contribution is small. Nonetheless the leading power correction can be modeled after integration over the final-state phase space as $[52,108,109]$

$$
\Delta \sigma_{\text {jettiness }}^{\mathrm{NNLO}}\left(\mathcal{T}_{0}^{\text {cut }}\right)=\Delta \sigma^{\mathrm{NNLO}}+c_{3} \frac{\mathcal{T}_{0}^{\text {cut }}}{Q} \log ^{3}\left(\frac{\mathcal{T}_{0}^{\text {cut }}}{Q}\right)+c_{2} \frac{\mathcal{T}_{0}^{\text {cut }}}{Q} \log ^{2}\left(\frac{\mathcal{T}_{0}^{\text {cut }}}{Q}\right)+\ldots
$$

where $Q$ is an appropriate hard scale of the process and $c_{2}, c_{3}$ are unknown constants. We have performed a fit of the results of our Monte-Carlo runs to this functional form of the $N$-jettiness NNLO coefficient for $Z Z$ and show the resulting fit as a black dotted line in figure 2. The fit allows us to numerically extract the value of the NNLO coefficient in the limit where $\mathcal{T}_{0} \rightarrow 0$. This value can be compared to the reconstructed NNLO coefficient for $Z Z$ production obtained in ref. [31],,$^{5}$ which is shown as a red line. We use the extrapolated value for our result for the $Z Z$ cross section at NNLO shown in table 2, which is in excellent agreement with the result $\sigma_{\mathrm{NNLO}}=16.91 \mathrm{pb}$ obtained in ref. [31].

\footnotetext{
${ }^{5}$ The NNLO coefficient was reconstructed by subtracting from the total NNLO $Z Z$ cross section quoted in table 1 of ref. [31] the NLO $Z Z$ cross section and the contribution from the loop-induced $g g$-channel.
} 


\begin{tabular}{|c|c|c|c|}
\hline & $\sigma_{\mathrm{LO}}[\mathrm{pb}]$ & $\sigma_{\mathrm{NLO}}[\mathrm{pb}]$ & $\sigma_{\mathrm{NNLO}}[\mathrm{pb}]$ \\
\hline Our Result & & & \\
MSWT2008 & $9.890_{-6.1 \%}^{+4.9 \%}$ & $14.508_{-2.4 \%}^{+3.0 \%}$ & $16.92_{-2.6 \%}^{+3.2 \%}$ \\
NNPDF3.0 & $9.845_{-6.2 \%}^{+5.2 \%}$ & $14.100_{-2.4 \%}^{+2.9 \%}$ & $16.69_{-2.8 \%}^{+3.1 \%}$ \\
\hline ATLAS [7] & \multicolumn{3}{|c|}{$17.3 \pm 0.6$ (stat.) \pm 0.5 (syst.) \pm 0.6 (lumi.) } \\
\hline CMS [8] & $17.2 \pm 0.5$ (stat.) \pm 0.7 (syst.) \pm 0.4 (theo.) $\pm 0.4($ lumi. $)$ \\
\hline
\end{tabular}

Table 2. Inclusive cross section for $Z Z$ production at the LHC run II $\sqrt{s}=13 \mathrm{TeV}$ at $\mathrm{LO}, \mathrm{NLO}$ and NNLO with $\mu_{R}=\mu_{F}=m_{Z}$, together with the measurements from ATLAS [7] and CMS [8]. Uncertainties in the theory calculation at each order are obtained by varying the renormalisation and factorisation scales in the range $0.5 m_{Z}<\mu_{R}, \mu_{F}<2 m_{Z}$ with the constraint $0.5<\mu_{F} / \mu_{R}<2$. Uncertainties in the experimental measurements denote absolute statistical, systematic and luminosity uncertainties.

As a consistency check we have also fitted a constant to the plateau region $\left(\mathcal{T}_{0}^{\text {cut }}<\right.$ $10^{-2} \mathrm{GeV}$ or $\mathcal{T}_{0}^{\text {cut }}<10^{-1} \mathrm{GeV}$ ) and these fits yield compatible results for $\Delta \sigma^{\mathrm{NNLO}}$. Further, we have also fitted the leading power corrections using (3.1) including only results for $\mathcal{T}_{0}^{\text {cut }}<1 \mathrm{GeV}$. When fitting the leading power corrections with $\mathcal{T}_{0}^{\text {cut }}<1 \mathrm{GeV}$ there is a strong correlation between $c_{3}$ and $Q$ as well as $c_{2}$ and $Q$; fixing $Q$ to values in the range $50-5000 \mathrm{GeV}$ we obtain compatible results for $\Delta \sigma^{\mathrm{NNLO}}$. Including in the fit results up to $\mathcal{T}_{0}^{\text {cut }}<10^{2} \mathrm{GeV}$, as shown in figure 2 , we obtain a stable fit also when $Q$ is treated as a free parameter.

The resulting theoretical predictions can be compared with the ATLAS and CMS measurements at $\sqrt{s}=13 \mathrm{TeV}[7,8]$, also shown in table 2 . In the same table we present an updated value for the NNLO cross section computed as described in the previous section using the more recently determined NNPDF-3.0 [106] PDF sets and an updated value for the $W$-boson mass of $M_{W}=80.385 \mathrm{GeV}$; these settings are also used for our phenomenological results in the following. We observe a significant improvement in the agreement with the data after the inclusion of the NNLO corrections.

In order to study in more detail the scale uncertainty of the cross section we present in figure 3 the renormalisation and factorisation scale dependence of the $Z Z$ cross section at LO, NLO and NNLO. The figure shows largely non-overlapping scale uncertainty bands which demonstrate that for this process, the scale variations are insufficient to estimate missing higher order terms in the perturbative expansion. This however is not unexpected since $Z Z$ production at the LHC is an electroweak process which exhibits no renormalisation scale dependence at LO. For this reason we obtain large NLO QCD corrections to the cross section which are outside the LO scale band. Moreover, when going from NLO to NNLO, the loop-induced gluon fusion channel $g g \rightarrow Z Z$ opens up, and due to the large gluon flux it represents a numerically significant contribution. Since this new channel contributes for the first time at NNLO its contribution cannot be captured by the scale variation of the NLO cross section. Therefore, when increasing the perturbative order, we can observe a systematic reduction of the factorisation scale dependence of the cross 


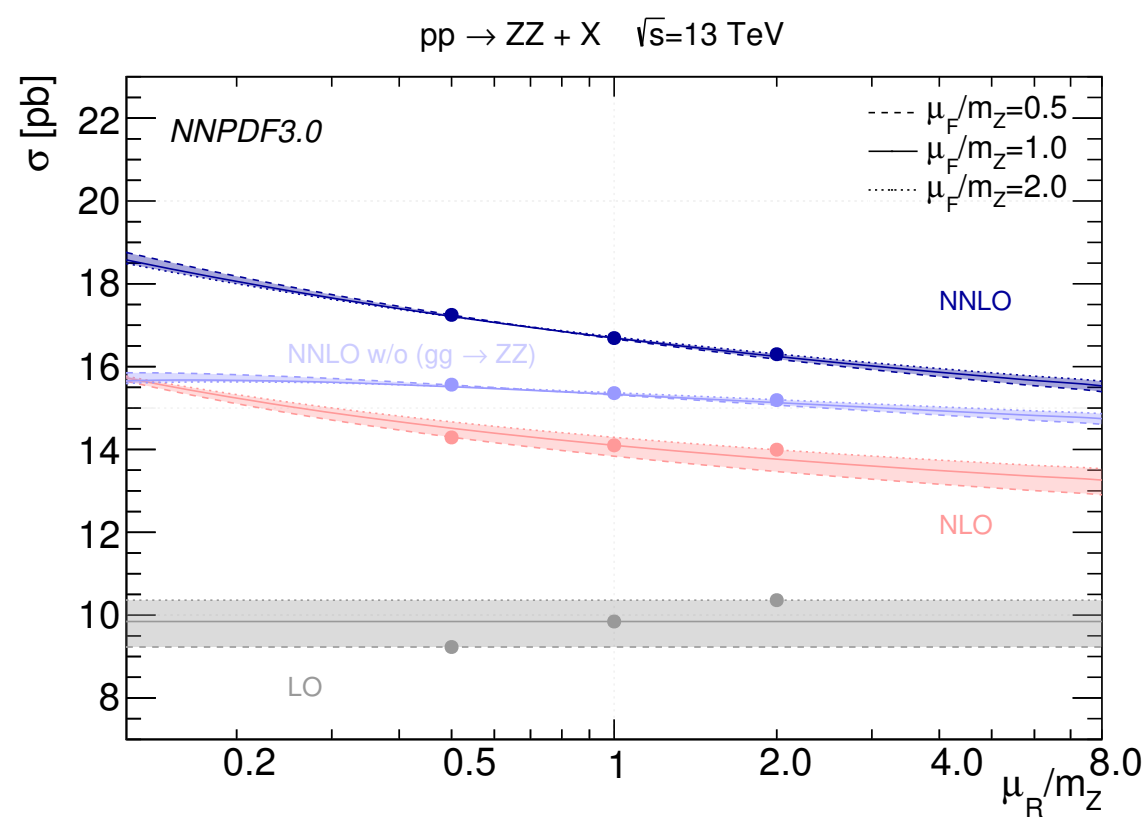

Figure 3. Renormalisation and factorisation scale dependence of the $Z Z$ cross section at LO, NLO and NNLO for the central scale choice $\mu_{R}=\mu_{F}=m_{Z}$ and with NNPDF-3.0 PDFs. We also show the NNLO result without the gluon fusion contributions. The thickness of the bands shows the variation in the cross section due to factorisation scale while the slope shows the renormalisation scale dependence. The scale uncertainty was obtained by varying the renormalisation and factorisation scales in the range $0.5 m_{Z}<\mu_{R}, \mu_{F}<2 m_{Z}$ with the constraint $0.5<\mu_{F} / \mu_{R}<2$.

section (indicated by the thickness of the scale uncertainty band), while there is no significant reduction of the renormalisation scale dependence. To show that this effect can be attributed to the gluon fusion channel opening up at NNLO, we also show the NNLO result excluding this channel, leading to an improved convergence of the perturbative expansion.

The appearance of new channels that open up at NNLO and their importance in the various kinematic regions can be studied by considering differential results. Due to the observed mild power corrections in this process we chose to fix the value of the 0-jettiness slicing parameter to $\mathcal{T}_{0}^{\text {cut }}=10^{-2} \mathrm{GeV}$ for all our histograms. In figure 4 we present the invariant mass of the $Z Z$ system and the average transverse momentum distribution $\left\langle p_{T, Z}\right\rangle$ of any $Z$-boson, defined as $\left\langle p_{T, Z}\right\rangle=\left(\left|p_{T}^{Z_{1}}\right|+\left|p_{T}^{Z_{2}}\right|\right) / 2$. We also present results for the loopinduced $g g \rightarrow Z Z$ channel.

In figure 4 a we show our results for the $Z Z$ invariant mass. In the first and second sub-panels we show the effect of the NLO and NNLO corrections, respectively. We observe in the first sub-panel large NLO QCD corrections which vary between $40 \%$ at low $m_{Z Z}$ and $60 \%$ at high $m_{Z Z}$, and change both the shape and normalisation of the predicted cross section with respect to the LO result. Going to NNLO we observe an approximately flat increase of the cross section of about $18 \%$ with respect to the NLO result, where approximately $60 \%$ of this effect comes from the loop-induced $g g \rightarrow Z Z$ channel, which is outside the scale uncertainty band of the NLO prediction. Similarly, in the transverse momentum distribution (figure 4b), we observe large NLO corrections of approximately 


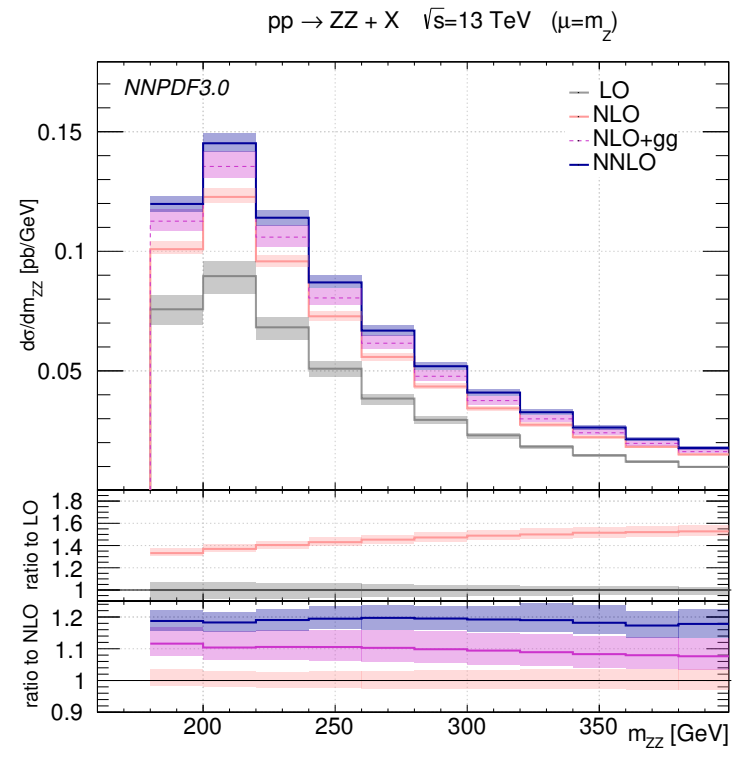

(a)

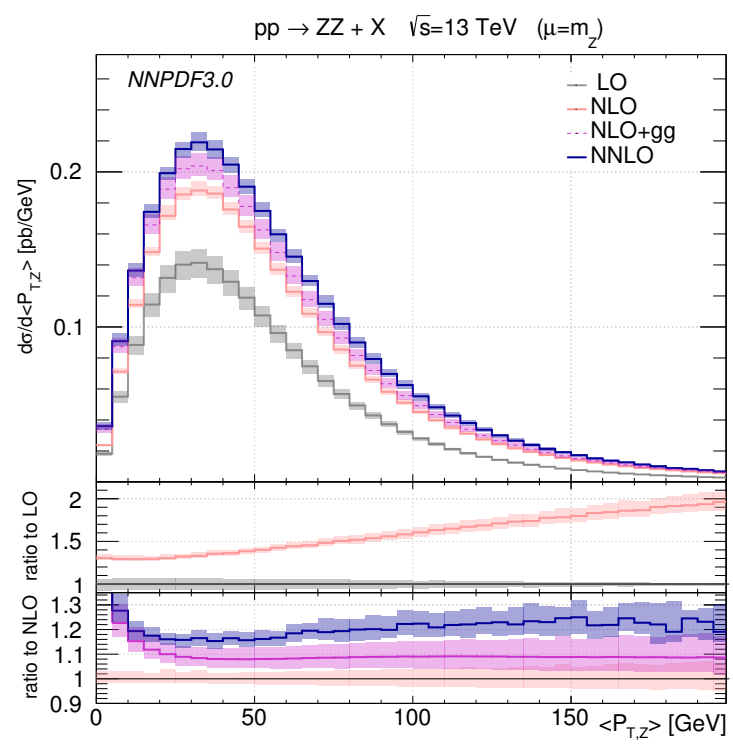

(b)

Figure 4. (a) $Z Z$ invariant mass distribution and (b) averaged transverse momentum distribution $\left\langle p_{T, Z}\right\rangle$ of the $Z$-bosons computed at LO, NLO and NNLO. In the two sub panels we show respectively the NLO/LO and NNLO/NLO $K$-factors to visualise the size of the higher order effects. The result for the contribution from the loop-induced $g g \rightarrow Z Z$ subset of the full NNLO correction is also shown separately. Shaded bands represent the theory uncertainty due to the variation of the factorisation and renormalisation scales.

$30 \%$ at low $\left\langle p_{T, Z}\right\rangle$, which can reach almost $100 \%$ at high $\left\langle p_{T, Z}\right\rangle$. The shape of the NNLO corrections in the second sub-panel largely follows the contribution of the loop-induced $g g \rightarrow Z Z$ channel at low $\left\langle p_{T, Z}\right\rangle$, and we observe a $30 \%$ effect at low $\left\langle p_{T, Z}\right\rangle$ which decreases to about $18 \%$ at high $\left\langle p_{T, Z}\right\rangle$. For both distributions we observe good convergence of the perturbative expansion, however the scale uncertainty bands do not overlap between the orders in the perturbative expansion that we have computed. These results show that the inclusion of NNLO effects in $Z Z$ production at the LHC is essential to obtain a reliable theoretical description of this process.

\section{Conclusions}

In this work we have calculated the NNLO QCD corrections to on-shell $Z$-boson pair production. Our calculation of the real emission contributions uses $N$-jettiness to isolate the infrared divergent contributions. We discussed our setup in some detail, also showing a comparison between results based on antenna subtraction and results based on $N$-jettiness for the NLO corrections.

After the inclusion of the NNLO correction in the theory prediction we found good agreement with the results of the recent ATLAS and CMS measurements. Due to the fact that the numerically sizeable loop-induced $g g \rightarrow Z Z$ contribution appears for the first time at NNLO, the scale uncertainties in the NNLO prediction are not reduced with respect to 
NLO. The NNLO corrections increase the NLO result by about $18 \%$, where almost $60 \%$ of this increase stems from the loop-induced $g g \rightarrow Z Z$ channel. In view of the numerical importance of this channel, it is desirable to add the two-loop diagrams, including massive top quark loops, to this channel, which will be left for a subsequent publication.

\section{Acknowledgments}

We thank Frank Tackmann, Simone Alioli and Max Stahlhofen for useful discussions on $N$-jettiness subtraction. This research was supported in part by the Research Executive Agency (REA) of the European Union under the Grant Agreement PITN-GA2012316704 (HiggsTools). We also acknowledge support by the Munich Institute for Astro- and Particle Physics (MIAPP) of the DFG cluster of excellence "Origin and Structure of the Universe". We are also grateful for support and resources provided by the Max Planck Computing and Data Facility (MPCDF).

Open Access. This article is distributed under the terms of the Creative Commons Attribution License (CC-BY 4.0), which permits any use, distribution and reproduction in any medium, provided the original author(s) and source are credited.

\section{References}

[1] ATLAS and CMS collaborations, Combination of results from the ATLAS and CMS experiments on anomalous triple gauge couplings in $Z Z$ production from $p p$ collisions at a centre-of-mass energy of $7 \mathrm{TeV}$ at the LHC, ATLAS-CONF-2016-036 (2016) [CMS-PAS-SMP-15-001] [inSPIRE].

[2] CMS collaboration, Measurement of $W^{+} W^{-}$and $Z Z$ production cross sections in $p p$ collisions at $\sqrt{s}=8 \mathrm{TeV}$, Phys. Lett. B 721 (2013) 190 [arXiv:1301.4698] [InSPIRE].

[3] CMS collaboration, Measurements of the $Z Z$ production cross sections in the $2 l 2 \nu$ channel in proton-proton collisions at $\sqrt{s}=7$ and $8 \mathrm{TeV}$ and combined constraints on triple gauge couplings, Eur. Phys. J. C 75 (2015) 511 [arXiv:1503.05467] [INSPIRE].

[4] ATLAS collaboration, Measurement of the $Z Z$ production cross section in proton-proton collisions at $\sqrt{s}=8 \mathrm{TeV}$ using the $Z Z \rightarrow \ell^{-} \ell^{+} \ell^{\prime} \ell^{\prime+}$ and $Z Z \rightarrow \ell^{-} \ell^{+} \nu \bar{\nu}$ channels with the ATLAS detector, JHEP 01 (2017) 099 [arXiv: 1610.07585] [INSPIRE].

[5] ATLAS collaboration, Measurement of the ZZ Production Cross Section in pp Collisions at $\sqrt{s}=13$ TeV with the ATLAS Detector, Phys. Rev. Lett. 116 (2016) 101801 [arXiv: 1512.05314] [INSPIRE].

[6] CMS collaboration, Measurement of the ZZ production cross section and $Z \rightarrow \ell^{+} \ell^{-} \ell^{\prime+} \ell^{\prime-}$ branching fraction in pp collisions at $\sqrt{s}=13$ TeV, Phys. Lett. B 763 (2016) 280 [Erratum ibid. B 772 (2017) 884] [arXiv:1607.08834] [INSPIRE].

[7] ATLAS collaboration, $Z Z \rightarrow \ell^{+} \ell^{-} \ell^{\prime+} \ell^{\prime-}$ cross-section measurements and search for anomalous triple gauge couplings in $13 \mathrm{TeV}$ pp collisions with the ATLAS detector, Phys. Rev. D 97 (2018) 032005 [arXiv: 1709.07703] [INSPIRE].

[8] CMS collaboration, Measurements of the $p p \rightarrow Z Z$ production cross section and the $Z \rightarrow 4 \ell$ branching fraction and constraints on anomalous triple gauge couplings at $\sqrt{s}=13$ TeV, Eur. Phys. J. C 78 (2018) 165 [arXiv: 1709.08601] [INSPIRE]. 
[9] ATLAS collaboration, Search for heavy $Z Z$ resonances in the $\ell^{+} \ell^{-} \ell^{+} \ell^{-}$and $\ell^{+} \ell^{-} \nu \bar{\nu}$ final states using proton-proton collisions at $\sqrt{s}=13 \mathrm{TeV}$ with the ATLAS detector, ATLAS-CONF-2017-058 (2017) [INSPIRE].

[10] J. Ohnemus and J.F. Owens, An Order- $\alpha_{s}$ calculation of hadronic $Z Z$ production, Phys. Rev. D 43 (1991) 3626 [INSPIRE].

[11] B. Mele, P. Nason and G. Ridolfi, QCD radiative corrections to $Z$ boson pair production in hadronic collisions, Nucl. Phys. B 357 (1991) 409 [inSPIRE].

[12] J. Ohnemus, Hadronic $Z Z, W^{-} W^{+}$and $W^{ \pm} Z$ production with $Q C D$ corrections and leptonic decays, Phys. Rev. D 50 (1994) 1931 [hep-ph/9403331] [InSPIRE].

[13] J.M. Campbell and R.K. Ellis, An Update on vector boson pair production at hadron colliders, Phys. Rev. D 60 (1999) 113006 [hep-ph/9905386] [INSPIRE].

[14] L.J. Dixon, Z. Kunszt and A. Signer, Vector boson pair production in hadronic collisions at $O\left(\alpha_{s}\right)$ : Lepton correlations and anomalous couplings, Phys. Rev. D 60 (1999) 114037 [hep-ph/9907305] [INSPIRE].

[15] D.A. Dicus, C. Kao and W.W. Repko, Gluon Production of Gauge Bosons, Phys. Rev. D 36 (1987) 1570 [INSPIRE].

[16] E.W.N. Glover and J.J. van der Bij, Z-boson pair production via gluon fusion, Nucl. Phys. B 321 (1989) 561 [INSPIRE].

[17] T. Matsuura and J.J. van der Bij, Characteristics of leptonic signals for $Z$ boson pairs at hadron colliders, Z. Phys. C 51 (1991) 259 [INSPIRE].

[18] C. Zecher, T. Matsuura and J.J. van der Bij, Leptonic signals from off-shell Z boson pairs at hadron colliders, Z. Phys. C 64 (1994) 219 [hep-ph/9404295] [INSPIRE].

[19] T. Binoth, N. Kauer and P. Mertsch, Gluon-induced QCD corrections to $p p \rightarrow Z Z \rightarrow \ell \bar{\ell} \ell^{\prime} \bar{\ell}^{\prime}$, in proceedings of the 16th International Workshop on Deep Inelastic Scattering and Related Subjects (DIS 2008), London, U.K., 7-11 April 2008, Science Wise Publishing, Berlin Germany (2008), p. 142 [arXiv: 0807.0024] [INSPIRE].

[20] J.M. Campbell, R.K. Ellis and C. Williams, Vector boson pair production at the LHC, JHEP 07 (2011) 018 [arXiv:1105.0020] [INSPIRE].

[21] N. Kauer, Interference effects for $H \rightarrow W W / Z Z \rightarrow \ell \bar{\nu}_{\ell} \bar{\ell}_{\ell}$ searches in gluon fusion at the LHC, JHEP 12 (2013) 082 [arXiv:1310.7011] [INSPIRE].

[22] F. Cascioli, S. Höche, F. Krauss, P. Maierhöfer, S. Pozzorini and F. Siegert, Precise Higgs-background predictions: merging NLO QCD and squared quark-loop corrections to four-lepton + 0,1 jet production, JHEP 01 (2014) 046 [arXiv:1309.0500] [INSPIRE].

[23] J.M. Campbell, R.K. Ellis and C. Williams, Bounding the Higgs width at the LHC using full analytic results for $g g \rightarrow e^{-} e^{+} \mu^{-} \mu^{+}$, JHEP 04 (2014) 060 [arXiv:1311.3589] [INSPIRE].

[24] N. Kauer, C. O'Brien and E. Vryonidou, Interference effects for $H \rightarrow W W \rightarrow \ell \nu q \bar{q}^{\prime}$ and $H \rightarrow Z Z \rightarrow \ell \bar{\ell} q \bar{q}$ searches in gluon fusion at the LHC, JHEP 10 (2015) 074 [arXiv: 1506.01694] [INSPIRE].

[25] C.S. Li, H.T. Li, D.Y. Shao and J. Wang, Soft gluon resummation in the signal-background interference process of $g g\left(\rightarrow h^{*}\right) \rightarrow Z Z$, JHEP 08 (2015) 065 [arXiv:1504.02388] [INSPIRE]. 
[26] T. Gehrmann, A. von Manteuffel, L. Tancredi and E. Weihs, The two-loop master integrals for $q \bar{q} \rightarrow V V$, JHEP 06 (2014) 032 [arXiv: 1404.4853] [INSPIRE].

[27] F. Caola, J.M. Henn, K. Melnikov and V.A. Smirnov, Non-planar master integrals for the production of two off-shell vector bosons in collisions of massless partons, JHEP 09 (2014) 043 [arXiv: 1404.5590] [INSPIRE].

[28] T. Gehrmann, A. von Manteuffel and L. Tancredi, The two-loop helicity amplitudes for $q \bar{q}^{\prime} \rightarrow V_{1} V_{2} \rightarrow 4$ leptons, JHEP 09 (2015) 128 [arXiv:1503.04812] [INSPIRE].

[29] A. von Manteuffel and L. Tancredi, The two-loop helicity amplitudes for $g g \rightarrow V_{1} V_{2} \rightarrow 4$ leptons, JHEP 06 (2015) 197 [arXiv: 1503.08835] [INSPIRE].

[30] F. Caola, J.M. Henn, K. Melnikov, A.V. Smirnov and V.A. Smirnov, Two-loop helicity amplitudes for the production of two off-shell electroweak bosons in gluon fusion, JHEP 06 (2015) 129 [arXiv: 1503.08759] [INSPIRE].

[31] F. Cascioli et al., ZZ production at hadron colliders in NNLO QCD, Phys. Lett. B 735 (2014) 311 [arXiv: 1405.2219] [INSPIRE].

[32] M. Grazzini, S. Kallweit and D. Rathlev, ZZ production at the LHC: fiducial cross sections and distributions in NNLO QCD, Phys. Lett. B 750 (2015) 407 [arXiv:1507.06257] [INSPIRE].

[33] F. Caola, K. Melnikov, R. Röntsch and L. Tancredi, QCD corrections to ZZ production in gluon fusion at the LHC, Phys. Rev. D 92 (2015) 094028 [arXiv: 1509.06734] [INSPIRE].

[34] F. Caola, M. Dowling, K. Melnikov, R. Röntsch and L. Tancredi, QCD corrections to vector boson pair production in gluon fusion including interference effects with off-shell Higgs at the LHC, JHEP 07 (2016) 087 [arXiv: 1605.04610] [INSPIRE].

[35] S. Alioli, F. Caola, G. Luisoni and R. Röntsch, ZZ production in gluon fusion at NLO matched to parton-shower, Phys. Rev. D 95 (2017) 034042 [arXiv:1609.09719] [InSPIRE].

[36] E. Accomando, A. Denner and A. Kaiser, Logarithmic electroweak corrections to gauge-boson pair production at the LHC, Nucl. Phys. B 706 (2005) 325 [hep-ph/0409247] [INSPIRE].

[37] A. Bierweiler, T. Kasprzik and J.H. Kühn, Vector-boson pair production at the LHC to $\mathcal{O}\left(\alpha^{3}\right)$ accuracy, JHEP 12 (2013) 071 [arXiv:1305.5402] [INSPIRE].

[38] J. Baglio, L.D. Ninh and M.M. Weber, Massive gauge boson pair production at the LHC: a next-to-leading order story, Phys. Rev. D 88 (2013) 113005 [Erratum ibid. D 94 (2016) 099902] [arXiv: 1307.4331] [INSPIRE].

[39] S. Gieseke, T. Kasprzik and J.H. Kühn, Vector-boson pair production and electroweak corrections in HERWIG++, Eur. Phys. J. C 74 (2014) 2988 [arXiv:1401.3964] [INSPIRE].

[40] B. Biedermann, Full NLO electroweak corrections to Z-boson pair production at the Large Hadron Collider, PoS (DIS2017) 168 [arXiv:1707.01029] [INSPIRE].

[41] B. Biedermann, A. Denner, S. Dittmaier, L. Hofer and B. Jäger, Electroweak corrections to $p p \rightarrow \mu^{+} \mu^{-} e^{+} e^{-}+X$ at the LHC: a Higgs background study, Phys. Rev. Lett. 116 (2016) 161803 [arXiv: 1601.07787] [INSPIRE].

[42] B. Biedermann, A. Denner, S. Dittmaier, L. Hofer and B. Jäger, Next-to-leading-order electroweak corrections to the production of four charged leptons at the LHC, JHEP 01 (2017) 033 [arXiv: 1611.05338] [INSPIRE]. 
[43] S. Catani and M. Grazzini, An NNLO subtraction formalism in hadron collisions and its application to Higgs boson production at the LHC, Phys. Rev. Lett. 98 (2007) 222002 [hep-ph/0703012] [INSPIRE].

[44] R. Boughezal, C. Focke, X. Liu and F. Petriello, $W$-boson production in association with a jet at next-to-next-to-leading order in perturbative QCD, Phys. Rev. Lett. 115 (2015) 062002 [arXiv: 1504.02131] [INSPIRE].

[45] J. Gaunt, M. Stahlhofen, F.J. Tackmann and J.R. Walsh, N-jettiness Subtractions for NNLO QCD Calculations, JHEP 09 (2015) 058 [arXiv: 1505.04794] [INSPIRE].

[46] K. Melnikov and M. Dowling, Production of two Z-bosons in gluon fusion in the heavy top quark approximation, Phys. Lett. B $\mathbf{7 4 4}$ (2015) 43 [arXiv: 1503.01274] [INSPIRE].

[47] J.M. Campbell, R.K. Ellis, M. Czakon and S. Kirchner, Two loop correction to interference in $g g \rightarrow Z Z$, JHEP 08 (2016) 011 [arXiv: 1605.01380] [INSPIRE].

[48] J. Gao, C.S. Li and H.X. Zhu, Top Quark Decay at Next-to-Next-to Leading Order in QCD, Phys. Rev. Lett. 110 (2013) 042001 [arXiv: 1210.2808] [INSPIRE].

[49] R. Boughezal, C. Focke, W. Giele, X. Liu and F. Petriello, Higgs boson production in association with a jet at NNLO using jettiness subtraction, Phys. Lett. B 748 (2015) 5 [arXiv: 1505. 03893] [INSPIRE].

[50] I.W. Stewart, F.J. Tackmann and W.J. Waalewijn, Factorization at the LHC: From PDFs to Initial State Jets, Phys. Rev. D 81 (2010) 094035 [arXiv:0910.0467] [InSPIRE].

[51] I.W. Stewart, F.J. Tackmann and W.J. Waalewijn, N-Jettiness: An Inclusive Event Shape to Veto Jets, Phys. Rev. Lett. 105 (2010) 092002 [arXiv: 1004.2489] [INSPIRE].

[52] I. Moult, L. Rothen, I.W. Stewart, F.J. Tackmann and H.X. Zhu, Subleading Power Corrections for N-Jettiness Subtractions, Phys. Rev. D 95 (2017) 074023 [arXiv: 1612.00450] [INSPIRE].

[53] W.T. Giele, E.W.N. Glover and D.A. Kosower, Higher order corrections to jet cross-sections in hadron colliders, Nucl. Phys. B 403 (1993) 633 [hep-ph/9302225] [INSPIRE].

[54] S. Alioli et al., Combining Higher-Order Resummation with Multiple NLO Calculations and Parton Showers in GENEVA, JHEP 09 (2013) 120 [arXiv: 1211.7049] [INSPIRE].

[55] S. Alioli, C.W. Bauer, C. Berggren, F.J. Tackmann, J.R. Walsh and S. Zuberi, Matching Fully Differential NNLO Calculations and Parton Showers, JHEP 06 (2014) 089 [arXiv:1311.0286] [INSPIRE].

[56] C.W. Bauer, S. Fleming and M.E. Luke, Summing Sudakov logarithms in $B \rightarrow X_{s} \gamma$ in effective field theory, Phys. Rev. D 63 (2000) 014006 [hep-ph/0005275] [INSPIRE].

[57] J.R. Gaunt, M. Stahlhofen and F.J. Tackmann, The Quark Beam Function at Two Loops, JHEP 04 (2014) 113 [arXiv:1401.5478] [InSPIRE].

[58] R. Kelley, M.D. Schwartz, R.M. Schabinger and H.X. Zhu, The two-loop hemisphere soft function, Phys. Rev. D 84 (2011) 045022 [arXiv:1105.3676] [INSPIRE].

[59] P.F. Monni, T. Gehrmann and G. Luisoni, Two-Loop Soft Corrections and Resummation of the Thrust Distribution in the Dijet Region, JHEP 08 (2011) 010 [arXiv:1105.4560] [INSPIRE].

[60] T. Becher and M. Neubert, Infrared singularities of scattering amplitudes in perturbative QCD, Phys. Rev. Lett. 102 (2009) 162001 [Erratum ibid. 111 (2013) 199905] [arXiv: 0901.0722] [INSPIRE]. 
[61] T. Becher and M. Neubert, On the Structure of Infrared Singularities of Gauge-Theory Amplitudes, JHEP 06 (2009) 081 [Erratum JHEP 11 (2013) 024] [arXiv:0903.1126] [INSPIRE].

[62] J.M. Campbell, R.K. Ellis, Y. Li and C. Williams, Predictions for diphoton production at the LHC through NNLO in QCD, JHEP 07 (2016) 148 [arXiv:1603.02663] [INSPIRE].

[63] S. Catani, L. Cieri, D. de Florian, G. Ferrera and M. Grazzini, Universality of transverse-momentum resummation and hard factors at the NNLO, Nucl. Phys. B 881 (2014) 414 [arXiv:1311.1654] [inSPIRE].

[64] S. Catani, The Singular behavior of QCD amplitudes at two loop order, Phys. Lett. B 427 (1998) 161 [hep-ph/9802439] [INSPIRE].

[65] J. Baglio et al., VBFNLO: A Parton Level Monte Carlo for Processes with Electroweak Bosons - Manual for Version 2.7.0, arXiv:1107.4038 [INSPIRE].

[66] F. Campanario, M. Kerner, L.D. Ninh and D. Zeppenfeld, Next-to-leading order QCD corrections to ZZ production in association with two jets, JHEP 07 (2014) 148 [arXiv: 1405.3972] [INSPIRE].

[67] J. Alwall et al., The automated computation of tree-level and next-to-leading order differential cross sections and their matching to parton shower simulations, JHEP 07 (2014) 079 [arXiv: 1405.0301] [InSPIRE].

[68] G. Cullen et al., Automated One-Loop Calculations with GoSam, Eur. Phys. J. C 72 (2012) 1889 [arXiv:1111.2034] [INSPIRE].

[69] G. Cullen et al., GoSam-2.0: a tool for automated one-loop calculations within the Standard Model and beyond, Eur. Phys. J. C 74 (2014) 3001 [arXiv:1404.7096] [InSPIRE].

[70] F. Cascioli, P. Maierhofer and S. Pozzorini, Scattering Amplitudes with Open Loops, Phys. Rev. Lett. 108 (2012) 111601 [arXiv:1111.5206] [INSPIRE].

[71] P. Nogueira, Automatic Feynman graph generation, J. Comput. Phys. 105 (1993) 279 [INSPIRE].

[72] J. Kuipers, T. Ueda, J.A.M. Vermaseren and J. Vollinga, FORM version 4.0, Comput. Phys. Commun. 184 (2013) 1453 [arXiv:1203.6543] [INSPIRE].

[73] G. Cullen, M. Koch-Janusz and T. Reiter, Spinney: A Form Library for Helicity Spinors, Comput. Phys. Commun. 182 (2011) 2368 [arXiv:1008.0803] [InSPIRE].

[74] P. Mastrolia, G. Ossola, T. Reiter and F. Tramontano, Scattering amplitudes from unitarity-based reduction algorithm at the integrand-level, JHEP 08 (2010) 080 [arXiv: 1006.0710] [INSPIRE].

[75] T. Binoth, J.P. Guillet, G. Heinrich, E. Pilon and T. Reiter, Golem95: A Numerical program to calculate one-loop tensor integrals with up to six external legs, Comput. Phys. Commun. 180 (2009) 2317 [arXiv: 0810.0992] [INSPIRE].

[76] G. Cullen et al., Golem95C: A library for one-loop integrals with complex masses, Comput. Phys. Commun. 182 (2011) 2276 [arXiv:1101.5595] [INSPIRE].

[77] J.P. Guillet, G. Heinrich and J.F. von Soden-Fraunhofen, Tools for NLO automation: extension of the golem95C integral library, Comput. Phys. Commun. 185 (2014) 1828 [arXiv: 1312.3887] [INSPIRE]. 
[78] H. van Deurzen, G. Luisoni, P. Mastrolia, E. Mirabella, G. Ossola and T. Peraro, Multi-leg One-loop Massive Amplitudes from Integrand Reduction via Laurent Expansion, JHEP 03 (2014) 115 [arXiv: 1312.6678] [inSPIRE].

[79] T. Peraro, Ninja: Automated Integrand Reduction via Laurent Expansion for One-Loop Amplitudes, Comput. Phys. Commun. 185 (2014) 2771 [arXiv:1403.1229] [InSPIRE].

[80] A. van Hameren, OneLOop: For the evaluation of one-loop scalar functions, Comput. Phys. Commun. 182 (2011) 2427 [arXiv:1007.4716] [INSPIRE].

[81] A. Gehrmann-De Ridder, T. Gehrmann and E.W.N. Glover, Antenna subtraction at NNLO, JHEP 09 (2005) 056 [hep-ph/0505111] [INSPIRE].

[82] E.W.N. Glover and J. Pires, Antenna subtraction for gluon scattering at NNLO, JHEP 06 (2010) 096 [arXiv: 1003 . 2824] [INSPIRE].

[83] J. Currie, E.W.N. Glover and S. Wells, Infrared Structure at NNLO Using Antenna Subtraction, JHEP 04 (2013) 066 [arXiv: 1301.4693] [INSPIRE].

[84] A. Daleo, T. Gehrmann and D. Maître, Antenna subtraction with hadronic initial states, JHEP 04 (2007) 016 [hep-ph/0612257] [inSPIRE].

[85] A. Daleo, A. Gehrmann-De Ridder, T. Gehrmann and G. Luisoni, Antenna subtraction at NNLO with hadronic initial states: initial-final configurations, JHEP 01 (2010) 118 [arXiv: 0912.0374] [INSPIRE].

[86] A. Gehrmann-De Ridder, T. Gehrmann and M. Ritzmann, Antenna subtraction at NNLO with hadronic initial states: double real initial-initial configurations, JHEP 10 (2012) 047 [arXiv:1207.5779] [INSPIRE].

[87] A. Gehrmann-De Ridder, T. Gehrmann, E.W.N. Glover, A. Huss and T.A. Morgan, The NNLO QCD corrections to $Z$ boson production at large transverse momentum, JHEP 07 (2016) 133 [arXiv: 1605. 04295] [INSPIRE].

[88] X. Chen, J. Cruz-Martinez, T. Gehrmann, E.W.N. Glover and M. Jaquier, NNLO QCD corrections to Higgs boson production at large transverse momentum, JHEP 10 (2016) 066 [arXiv: 1607.08817] [INSPIRE].

[89] J. Currie, E.W.N. Glover and J. Pires, Next-to-Next-to Leading Order QCD Predictions for Single Jet Inclusive Production at the LHC, Phys. Rev. Lett. 118 (2017) 072002 [arXiv: 1611.01460] [INSPIRE].

[90] J. Currie, A. Gehrmann-De Ridder, T. Gehrmann, E.W.N. Glover, A. Huss and J. Pires, Precise predictions for dijet production at the LHC, Phys. Rev. Lett. 119 (2017) 152001 [arXiv: 1705.10271] [INSPIRE].

[91] M. Czakon, A novel subtraction scheme for double-real radiation at NNLO, Phys. Lett. B 693 (2010) 259 [arXiv:1005.0274] [InSPIRE].

[92] R. Boughezal, F. Caola, K. Melnikov, F. Petriello and M. Schulze, Higgs boson production in association with a jet at next-to-next-to-leading order in perturbative QCD, JHEP 06 (2013) 072 [arXiv:1302.6216] [INSPIRE].

[93] M. Czakon and D. Heymes, Four-dimensional formulation of the sector-improved residue subtraction scheme, Nucl. Phys. B 890 (2014) 152 [arXiv:1408.2500] [InSPIRE].

[94] F. Caola, K. Melnikov and R. Röntsch, Nested soft-collinear subtractions in NNLO QCD computations, Eur. Phys. J. C 77 (2017) 248 [arXiv:1702.01352] [INSPIRE]. 
[95] V. Del Duca, C. Duhr, G. Somogyi, F. Tramontano and Z. Trócsányi, Higgs boson decay into b-quarks at NNLO accuracy, JHEP 04 (2015) 036 [arXiv: 1501.07226] [INSPIRE].

[96] G. Somogyi, A. Kardos, Z. Ször and Z. Trócsányi, Higher order corrections in the CoLoRFulNNLO framework, Acta Phys. Polon. B 48 (2017) 1195 [arXiv:1706.01688] [INSPIRE].

[97] R. Bonciani, S. Catani, M. Grazzini, H. Sargsyan and A. Torre, The $q_{T}$ subtraction method for top quark production at hadron colliders, Eur. Phys. J. C 75 (2015) 581 [arXiv: 1508.03585] [INSPIRE].

[98] R. Boughezal et al., Z-boson production in association with a jet at next-to-next-to-leading order in perturbative QCD, Phys. Rev. Lett. 116 (2016) 152001 [arXiv:1512.01291] [INSPIRE].

[99] J.M. Campbell, R.K. Ellis and C. Williams, Associated production of a Higgs boson at NNLO, JHEP 06 (2016) 179 [arXiv:1601.00658] [INSPIRE].

[100] R. Boughezal, X. Liu and F. Petriello, W-boson plus jet differential distributions at NNLO in QCD, Phys. Rev. D 94 (2016) 113009 [arXiv: 1602.06965] [INSPIRE].

[101] R. Boughezal, X. Liu and F. Petriello, Phenomenology of the Z-boson plus jet process at NNLO, Phys. Rev. D 94 (2016) 074015 [arXiv: 1602.08140] [InSPIRE].

[102] R. Boughezal et al., Color singlet production at NNLO in MCFM, Eur. Phys. J. C 77 (2017) 7 [arXiv: 1605. 08011] [INSPIRE].

[103] J.M. Campbell, R.K. Ellis and C. Williams, Direct Photon Production at Next-to-Next-to-Leading Order, Phys. Rev. Lett. 118 (2017) 222001 [arXiv:1612.04333] [INSPIRE].

[104] J.M. Campbell, R.K. Ellis and C. Williams, Driving missing data at the LHC: NNLO predictions for the ratio of $\gamma+j$ and $Z+j$, Phys. Rev. D 96 (2017) 014037 [arXiv: 1703.10109] [INSPIRE].

[105] A.D. Martin, W.J. Stirling, R.S. Thorne and G. Watt, Update of parton distributions at NNLO, Phys. Lett. B 652 (2007) 292 [arXiv:0706.0459] [INSPIRE].

[106] NNPDF collaboration, R.D. Ball et al., Parton distributions for the LHC Run II, JHEP 04 (2015) 040 [arXiv: 1410.8849] [INSPIRE].

[107] A. Buckley et al., LHAPDF6: parton density access in the LHC precision era, Eur. Phys. J. C 75 (2015) 132 [arXiv:1412.7420] [INSPIRE].

[108] R. Boughezal, X. Liu and F. Petriello, Power Corrections in the N-jettiness Subtraction Scheme, JHEP 03 (2017) 160 [arXiv: 1612.02911] [INSPIRE].

[109] I. Moult, L. Rothen, I.W. Stewart, F.J. Tackmann and H.X. Zhu, N-jettiness subtractions for $g g \rightarrow H$ at subleading power, Phys. Rev. D 97 (2018) 014013 [arXiv:1710.03227] [INSPIRE]. 